\title{
Correction for beam divergence effect in a NRSE spectrometer with high resolution
}

\section{AUTHOR(S):}

Kitaguchi, Masaaki; Hino, M; Kawabata, Y; Hayashida, H; Tasaki, S; Maruyama, R; Ebisawa, T

\section{CITATION:}

Kitaguchi, Masaaki ...[et al]. Correction for beam divergence effect in a NRSE spectrometer with high resolution. Measurement Science and Technology 2008, 19(3): 034014.

\section{ISSUE DATE:}

2008-03-01

URL:

http://hdl.handle.net/2433/108301

\section{RIGHT:}

This is an author-created, un-copyedited version of an article accepted for publication in Measurement Science and Technology. IOP Publishing Ltd is not responsible for any errors or omissions in this version of the manuscript or any version derived from it. The definitive publisher authenticated version is available online at

http://dx.doi.org/10.1088/0957-0233/19/3/034014; This is not the published version. Please cite only the published version.; この論文は出版社版でありません。引用の際には出版社版をご確認ご利用ください。 


\title{
Correction for beam divergence effect in NRSE spectrometer with high resolution
}

\author{
M Kitaguchi ${ }^{1}$, M Hino ${ }^{1}$, Y. Kawabata ${ }^{1}$, H Hayashida $^{2}$, S \\ Tasaki $^{2}, \mathbf{R}$ Maruyama ${ }^{3}$ and T Ebisawa ${ }^{3}$ \\ ${ }^{1}$ Research Reactor Institute, Kyoto University, Kumatori, Osaka, 590-0494, Japan, \\ ${ }^{2}$ Department of Nuclear Engineering, Kyoto University, Kyoto, 606-8501, Japan, \\ ${ }^{3}$ JAEA, Tokai, Ibaraki, 319-1195, Japan, \\ E-mail: kitaguch@rri.kyoto-u.ac.jp
}

\begin{abstract}
Correction for beam divergence effect in neutron resonance spin echo (NRSE) spectrometer will be discussed. The new type of devices called "coupling coils" is proposed to change the spin quantization axis for the correction. The feasibility of correction for the vertical divergence has been demonstrated by numerical calculations and Monte-Carlo simulations.
\end{abstract}

PACS numbers: 03.75.Be, 03.75.Dg, 61.12.Ex

Keywords: neutron resonance spin echo, correction for beam divergence effect Submitted to: Meas. Sci. Techno. 


\section{Introduction}

Neutron spin echo (NSE) is one of the techniques with the highest energy resolution for quasi-elastic scattering [1]. The difference between incident and scattered neutron velocity is measured as the difference between the spin precession provided by static magnetic fields before and after the sample. The energy resolution is limited by the deviation of the precession due to the inhomogeneity of the magnetic fields and the divergent beam because the precession is proportional to the field integral. Correction of the precession for the "beam divergence effect" is needed in order to keep the neutron intensity by taking the divergent beam. One of the devices for the correction is "Fresnel coil." The arrangement of Fresnel coils enable us to perform the measurement with the Fourier time up to 400 ns $[2,3,4,5]$.

Neutron resonance spin echo (NRSE) spectrometer, which is a variety of neutron spin echo, contains resonance spin flippers (RSFs) and zero-fields instead of homogeneous static magnetic fields for spin precession in the conventional NSE [6]. It has the advantage to be constructed in small area and to perform measurements with less sensitivity of magnetic environment. In the case of NRSE, the energy resolution is limited mainly by the effect of the divergent beam. The beam divergence makes the deviation of the relative phase between up- and down-spin components, which is equivalent to spin precession, because the relative phase is proportional to the neutron flight length. Some methods to correct the beam divergence effect for NRSE have been proposed and demonstrated [7, 8, 9], however, they can not be applied for high resolution NRSE spectrometer. Now we discuss on the correction for much higher resolution NRSE by using the smooth coupling the Fresnel-like fields to RSFs.

\section{Correction in NRSE}

The relative phase between the two spin components is proportional to the neutron flight length and the difference of wavenumber between the components. For the beam with the incident angle of $\theta$, the length is changed from $L$ to $L / \cos \theta$, where $L$ is the distance between the two RSFs. The relative phase becomes

$$
\phi=\Delta k L / \cos \theta,
$$

where $\Delta k$ is the difference of wavenumber. The deviation of the phase due to the distribution of the incident angle in the beam can be corrected by using three magnetic fields with the strength which is proportional to the square of the radius. In the conventional NSE, the representation of the $\theta$-dependence of the spin precession is similar to the equation (1). A Fresnel coil makes the magnetic field with the strength according to $r^{2}$. The beam divergence effect can be successfully corrected with the arrangement of three Fresnel coils in the static magnetic field of NSE (figure 1).

Some modifications of the arrangement of Fresnel coils are required in order to be applied for NRSE. In NSE, the spin quantization axis is parallel to the direction of the neutron beam. The direction of the correction fields which make the additional field 


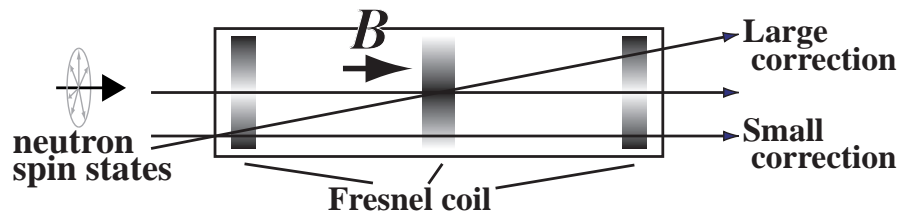

Figure 1. Concept of Fresnel coils. Black arrow shows spin quantization axis. Spin precession is in the plane perpendicular to the axis (gray arrows). Neutrons with larger incident angle are added larger correction.

integral is also along to the beam. The magnetic field with the strength depending on the radius and with the direction along the beam can be provided by the Fresnel coil. However, in NRSE spectrometer, the spin quantization axis is generally transverse to the beam. The vertical magnetic fields in RSFs is required for the bootstrap arrangement for high resolution NRSE [10]. The spin quantization axis need to be changed adiabatically from the vertical direction of RSFs into the longitudinal direction of Fresnel coils in zerofield between the RSFs. The magnetic field for the transition of the spin quantization axis makes the vertical asymmetric distribution of the field integral. When neutrons pass through the two transition fields at the entrance and the exit of the weak field between the RSFs, the relative phase is distributed depending on the difference of the field integral (figure 2). Although the weak guide field between the RSFs, or zero field for ideal case, is required in NRSE, it is difficult to utilize the Fresnel coils due to the stray field.

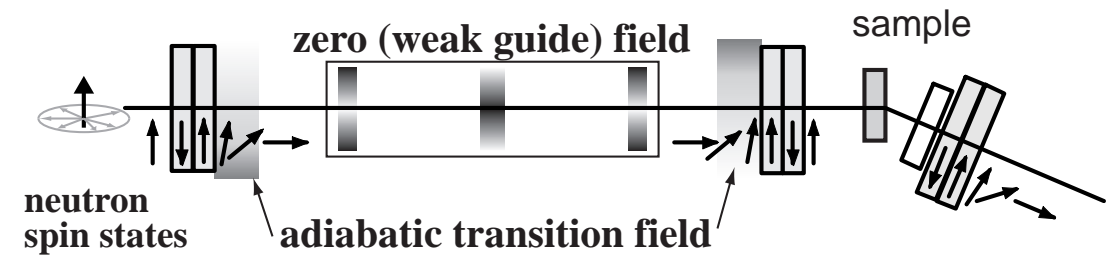

Figure 2. Fresnel coils in NRSE. Gray gradation area expresses the distribution of the field strength. Transition of spin state before and after very weak guide field makes deviation of the relative phase.

\section{Coupling coils for correction}

Now we consider the devices which make the transition of spin quantization axis only at the RSFs. The Fresnel-like distribution of the correction field can be applied because of the longitudinal quantization axis in the other area. The quantization axis of the incident neutrons changes from longitudinal direction into vertical direction just before a RSF. After the flip of the spin, the quantization axis changes to longitudinal direction. We call the devices which provides the transition field as "coupling coils" (figure 3). Neutron polarization is guided from the longitudinal magnetic field into the vertical magnetic field in the coupling coils adiabatically, after then, goes into the vertical field of the RSF non-adiabatically. 
Coupling coils consist of a longitudinal solenoid and a vertical solenoid. Mu-metal box covers the vertical coil in order to enclose magnetic flux. One side of the vertical solenoid is opened for incident beam. The magnetic field provided by the longitudinal coil and the field by the vertical coil are connected smoothly. The numerical calculation tells us that the field integral of the transition field along the beam flight path is proportional to the square of the vertical position with the vertically shifted center of the parabola. The field integral is written as

$$
\int_{\text {beforeRSF }} B d z=a\left(y-y_{0}\right)^{2}+b,
$$

where $y_{0}$ is the shift of the center, $a$ and $b$ are constants. By using a set of the coupling coils for "transition for correction," the vertical shift of the center of the field integral provides the distributed relative phase for the vertical divergence of the beam. The second coupling coils, which are symmetrical arrangement of the first coils, is placed just after the RSF to provide the transition field with the parabola field integral. The vertical shift of the center of the parabola is reverse to the first one, like as

$$
\int_{\text {afterRSF }} B d z=a\left(y+y_{0}\right)^{2}+b .
$$

The total field integral through the RSF with spin-flipping is proportional to the square of the vertical position without the shift of the center of the parabola. It can be written as

$$
\int_{\text {throughRSF }} B d z=2 a y^{2}+\left(2 a y_{0}^{2}+2 b\right),
$$

which is the same representation as the correction field only for the vertical divergence. The magnetic field functions not only as the "transition for RSFs" but also as a "vertical Fresnel coil." A pair of RSFs with the coupling coils at the entrance and the exit of the zero field in the NRSE spectrometer can replace the two Fresnel coils as the correction devices.

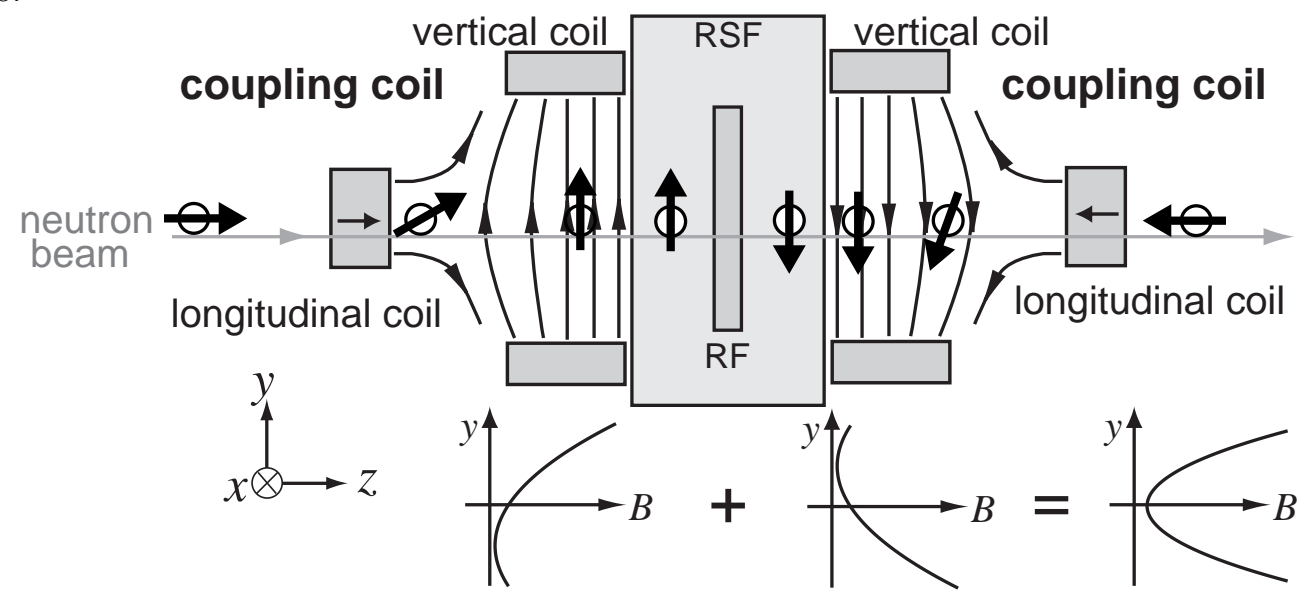

Figure 3. Concept of coupling coils. Coupling coils change the spin quantization axis to vertical only at RSFs. Fine arrows show magnetic fields with the coils. The arrows on circles represent spin quantization axis. Spin precession is in the plane perpendicular to the arrows. The graphs show field integral at coupling coils and the sum of them. Two set of coupling coils function as vertical Fresnel coil. 


\section{Simulation}

In order to demonstrate the feasibility of the correction by using the coupling coils, the magnetic fields provided by the coils were calculated and the behavior of neutron spin was estimated with Monte-Carlo simulations. We have only to calculate for the first arm of the spectrometer because the relative phase must be uniform for divergent beam in the first arm and in the second arm respectively. In this calculation, only vertical divergence was considered. All devices in the system for the calculation was prepared as three dimensional elements. Magnetic field of the each point were calculated also as a three dimensional vectors. The calculation of the field integral and the simulation of the spin behavior were considered in the center region of the beam width because the each device was wide enough to enable us to assume the symmetry of the magnetic field for the beam. We also assumed that the flipping probability for all RSFs was always one and that the relative phase added at the flipping at the RSFs was always zero. The external field including earth's field was neglected.

The coupling coils were made with the wires with the cross section of $1 \mathrm{~mm}$ square. A rectangle solenoid was for the longitudinal coil in a set of coupling coils. Another rectangle solenoid with the opening of the height of $50 \mathrm{~mm}$, which was made by bending of the wires, provided the vertical field with leak for transition field. Mu-metal boxes were made with the plates of thickness of $1 \mathrm{~mm}$. The configuration of a coupling coil is illustrated in figure 4 .

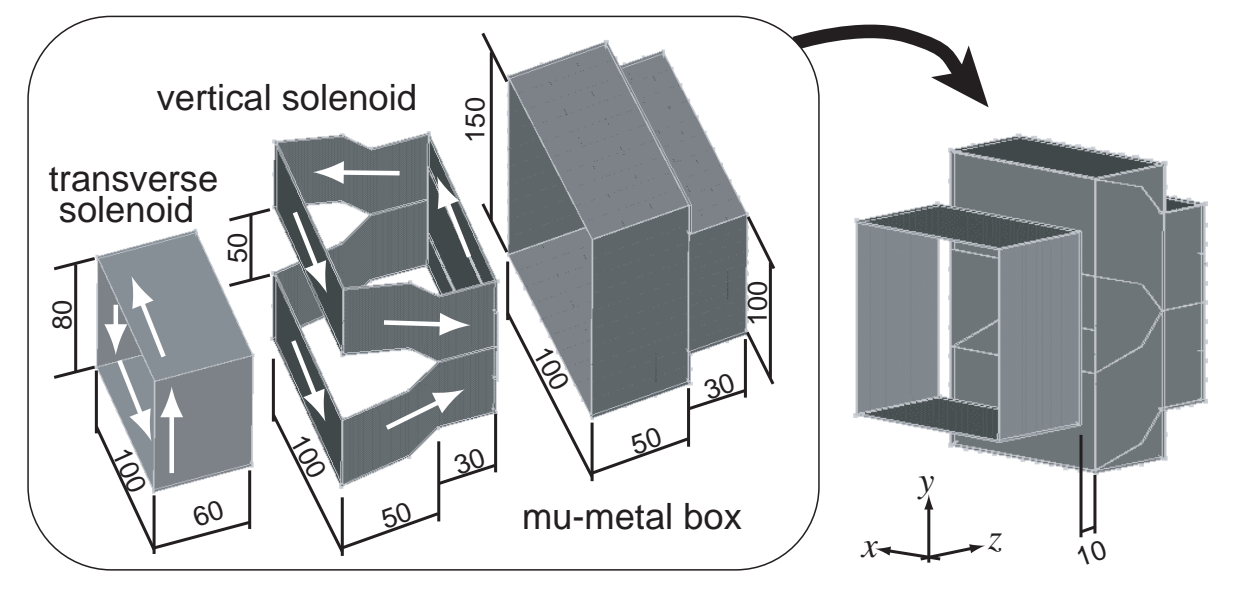

Figure 4. Coupling coils. The vertical solenoid is in the mu-metal box. White arrows shows the current for the coils.

Tri-bootstrap arrangement is an idea to apply RSFs to the correction with the coupling coils. The correction with the coupling coils requires the odd times of spin-flip at the RSFs. In the original idea of bootstrap arrangement, the combination of two same RSFs encloses the strong magnetic flux for the resonance condition [10]. In the tri-bootstrap arrangement the combination of three RSFs encloses the magnetic flux by the center RSF with double of the cross section of the flux (figure 5). It also gives us the higher effective frequency of NRSE spectrometer. 

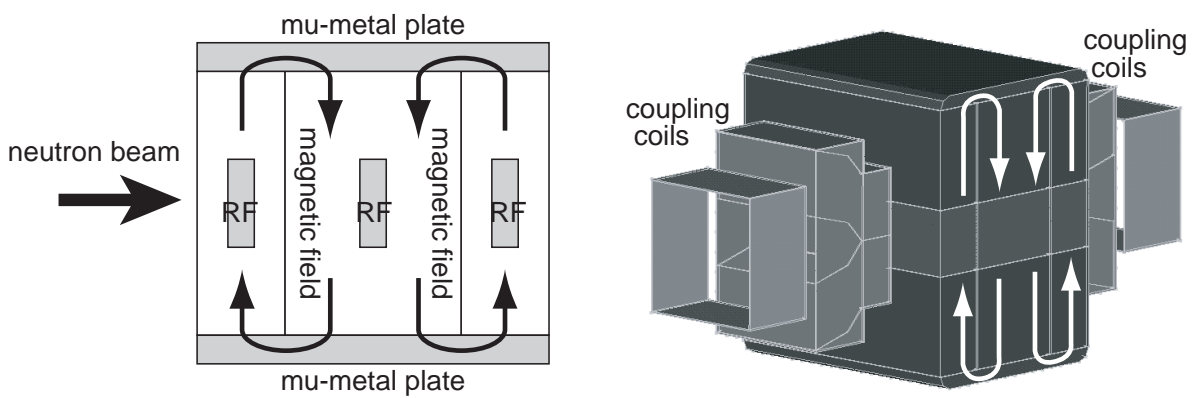

Figure 5. Tri-bootstrap arrangement of RSFs.

Although the first and the third of three Fresnel coils for the correction in NSE were replaced with the coupling coils, the center Fresnel coil was still required in this arrangement using the coupling coils. A solenoidal coil with the small radius can function as the correction device instead of the Fresnel coil for NRSE only with the longitudinal magnetic fields because the coil provides the longitudinal field with the strength which is proportional to $r^{2}[11]$. In this system the rectangle solenoid with the narrow height was utilized to make correction field with the strength which was proportional to $y^{2}$. The height of beam area was $50 \mathrm{~mm}$ and the width was $100 \mathrm{~mm}$. The length of the coil was $80 \mathrm{~mm}$. The wire was winded $80 \times 10=800$ turns. It was made with the wires with the cross section of $1 \mathrm{~mm}$ square.

The magnetic field in the system including two RSFs and four set of coupling coils, which illustrated in figure 6 , is calculated with integral element method by ELF/MAGIC (c) [12]. When the field is strong or the change of the strength is large, the calculation was performed every $0.1 \mathrm{~mm}$. Otherwise the magnetic field was calculated every $1 \mathrm{~mm}$. The calculated magnetic fields of some points around the coupling coils is shown in figure 7 . The parameters for calculation is shown in table 1.

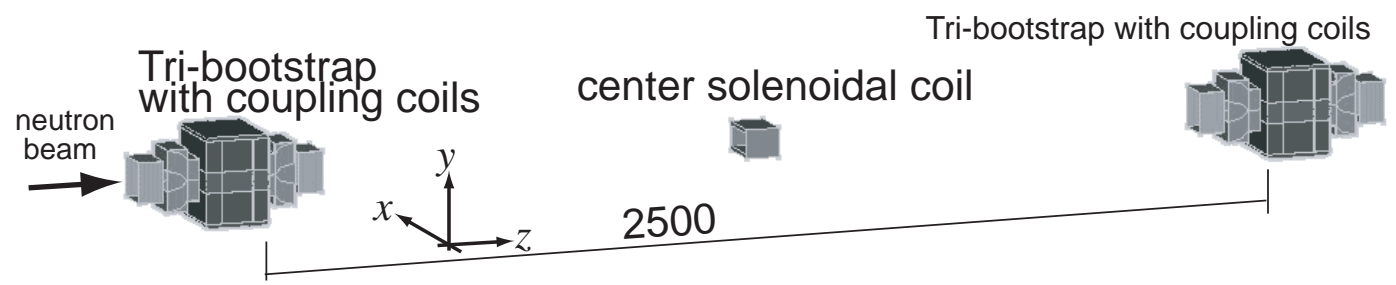

Figure 6. System for calculation.

Ten thousand neutrons with the wavelength of $2 \mathrm{~nm}$ were injected into the system. There were two slits of the height of $40 \mathrm{~mm}$ at the both ends of the system, which had the distance of $3390 \mathrm{~mm}$. The maximum vertical divergence angle of the beam was 0.0236 rad. The relative phase between up- and down-component, which is corresponding to the spin precession, was calculated with the field integral of the calculation points through the neutron pass.

We considered that the frequency of each RSF of tri-bootstrap arrangement was 2 $\mathrm{MHz}$. This leaded the Fourier time of $1.6 \mu \mathrm{s}$. 


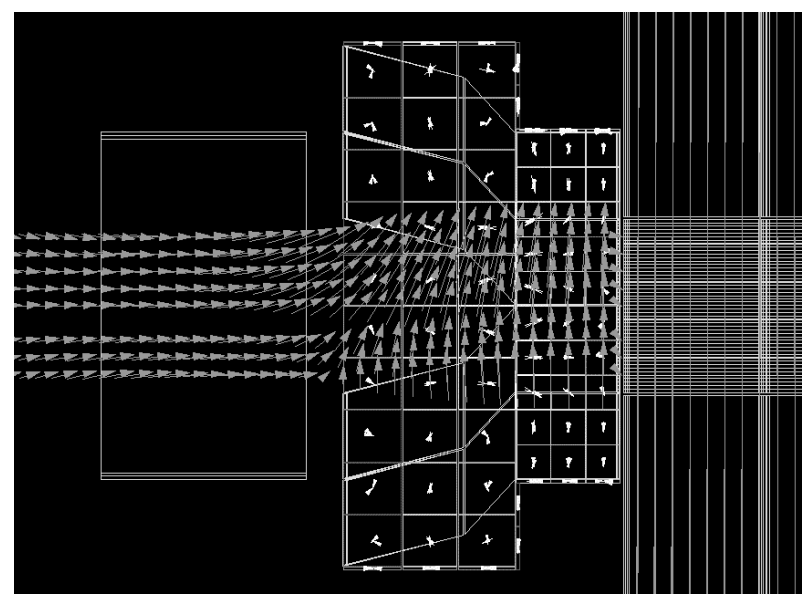

Figure 7. Magnetic field around the coupling coils (Side view). Neutrons come from left side of this figure. Gray arrows indicate the magnetic fields. White arrows show the magnetization of the mu-metal box. Gray lines and boxes show the mesh for the calculation of the field.

Table 1. Parameters for calculation

\begin{tabular}{llccc}
\hline & & $\begin{array}{c}\text { vertical } \\
\text { solenoid }\end{array}$ & $\begin{array}{c}\text { transverse } \\
\text { solenoid }\end{array}$ & $\begin{array}{c}\text { center } \\
\text { solenoid }\end{array}$ \\
\hline current & {$[\mathrm{A}]$} & 3.8 & 8.8 & 2.475 \\
length & {$[\mathrm{mm}]$} & 100 & 60 & 80 \\
turn & & 100 & 60 & 800 \\
\hline
\end{tabular}

\section{Results and discussion}

The relative phase of each neutron through the system was calculated individually as the sum of the phase according to the equation (1) and the additional phase by the field integral by the coupling coils. We can get the informations about both of the incident angle and the relative phase for every neutrons. Figure 8 shows the relative phase distribution for the transverse momentum of neutrons $k_{y}=k \sin \theta$, where $k$ is the momentum of the neutrons. Without the correction, the parabolic distribution according to $L / \cos \theta$ was provided (gray points). The distribution was reduced by the correction with the coupling coils (black points). The histograms of the relative phase is shown in figure 9. We find clearly that the broad distribution without the correction shrinks into the sharp peak by using the correction coils.

The depolarization due to the transition field by the coupling coils was also simulated based on Schrödinger equation. The incident neutrons with the longitudinal polarization, which was parallel to the spin quantization axis at the entrance of the system, were considered. The changes of the spinor through the small regions for the calculation of magnetic field were traced by quantum mechanics step by step. The histogram of the longitudinal component of the neutron spin at the exit of the system is shown in figure 10. The average polarization through the system was 0.93. The 


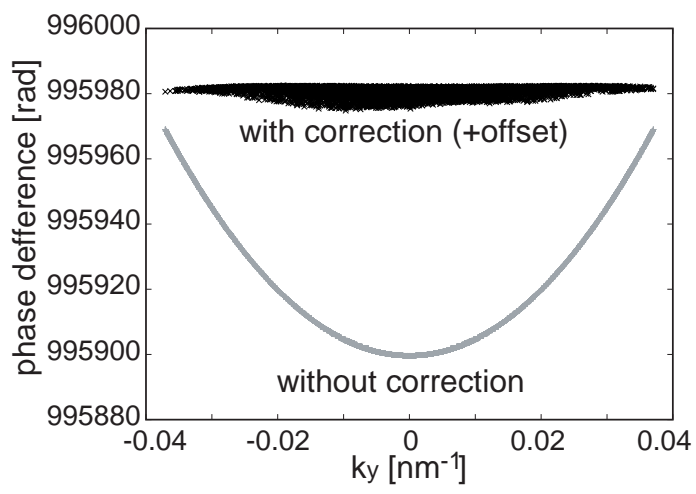

Figure 8. Phase distribution for the vertical moment. For the relative phase with the correction the constant value is added to fit the data points into the frame.

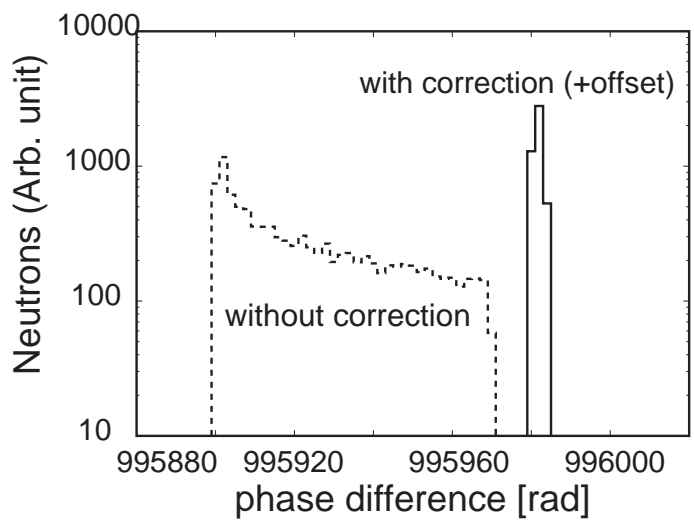

Figure 9. Histogram of phase distribution. For the relative phase with the correction the constant value is added to fit the data points into the frame.

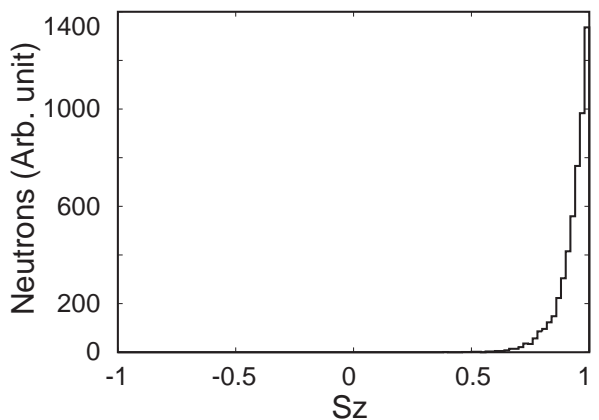

Figure 10. Polarization for the longitudinal component through the system. The incident neutrons were perfectly polarized into the longitudinal direction.

depolarization was occurred in the connection area between the field from the coupling coils and the reverse field from the center solenoid. A Fresnel coil which can make the magnetic field distribution for the center has the advantage to decrease the reverse field in order to reduce the depolarization. 
The above are not the results with the optimized conditions for the shapes of the coils, the distances between the devices, and the electric current. We can control the transition field by changing the current of coils even after installing the devices. The optimum conditions must be studied for the practical use.

The system and the magnetic field in this simulation was symmetric for the horizontal direction. The correction for the horizontal divergence can be performed by using the "horizontal Fresnel coils." That can be realized by using solenoids with the narrow width, for example. The center rectangle solenoid in this simulation will be replaced with the normal Fresnel coil. Three dimensional analysis for the correction for the beam divergence effect is also required.

\section{Conclusions}

We demonstrated the feasibility of correction for the beam divergence effect in high resolution NRSE spectrometer by the numerical calculation and Monte-Carlo simulation. The coupling coils, which change the spin quantization axis into vertical direction from longitudinal one only at resonance spin flippers, also provide the magnetic fields for the correction of vertical divergence effect. We are continuing to optimize the design of the coupling coils and the simulation. Now we are planning to construct resonance spin echo spectrometers in pulsed neutrons like J-PARC [13]. The RSF with high frequency has been developed for the NRSE with pulsed neutrons [14]. Whole simulation through the total system of the NRSE spectrometers is in progress. We are planning experiments to confirm the phase correction with the coils.

\section{Acknowledgments}

This work was supported by the inter-university program for common use JAEA and KUR, and financially by the program of Development of System and Technology for Advanced Measurement and Analysis (SENTAN), JST and by a Grant-in-Aid for Scientific Research No. 17760681 and No. 17760055 of JSPS.

\section{References}

[1] Mezei F 1972 Neutron spin echo: A new concept in polarized thermal neutron techniques $Z$. Phys. 255146

[2] Zeyen C M E, Rem P C, Hartmann R A and van de Klundert L J M 1988 Optimal neutron Larmor precession magnets IEEE Trans. Magn. 241540

[3] Monkenbusch M 1990 On solenoid design for neutron spin echo spectrometers Nucl. Instr. and Meth. A 287465

[4] Monkenbusch M, Schätzler R and Richter D 1997 The Jülich neutron spin-echo spectrometer Design and performance Nucl. Instr. and Meth. A 399301

[5] Schleger P et al 1999 The sub-neV resolution NSE spectrometer IN15 at the Institute LaueLangevin Physica B 26649

[6] Gähler R and Golub R 1987 A high resolution neutron spectrometer for quasielastic scattering on the basis of spin-echo and magnetic resonance Z. Phys. B 65269 
[7] Monkenbusch M 1999 Correction scheme for divergent beams in zero-field spin-echo spectrometers Nucl. Instr. Meth. A 437455

[8] Bleuel M et al 2002 Neutron Spin Echo Spectroscopy, Basics, Trends and Applications, Lecture Notes in Physics Vol 601 (Berlin:Springer) p 176

[9] Häussler W Schmidt U Ehlers G Mezei F 2003 Neutron resonance spin echo using spin echo correction coils Chem. Phys. 292501

[10] Köppe M et al 1999 Prospects of resonance spin echo Physica B 26675

[11] Maruyama R Ebisawa T Hino M Tasaki S and Kawabata Y 2004 Simulations on a beam divergence correction coil for neutron resonance spin echo Nucl. Instr. and Meth. A 529213

[12] ELF Corporation http://www.elf.co.jp

[13] Kawabata Y Hino M Kitaguchi M Hayashida H Tasaki S Ebisawa T Yamazaki D Maruyama R Seto H Nagao M and Kanaya T 2006 Neutron resonance spin echo and MIEZE spectrometer development project in Japan Physica B 385-386 1122

[14] Hayashida H Kitaguchi M Hino M Kawabata Y Maruyama R Ebisawa T 2007 Development of a resonance spin flipper for NRSE/MIEZE on a pulsed neutron beam with an oscillating frequency of $500 \mathrm{kHz}$ Nucl. Instrm. Meth. A $\mathbf{5 7 4} 292$ 